\title{
FROBENIUS-SCHUR INDICATORS OF CHARACTERS IN BLOCKS WITH CYCLIC DEFECT
}

\author{
JOHN C. MURRAY
}

\begin{abstract}
Let $p$ be an odd prime and let $B$ be a $p$-block of a finite group which has cyclic defect groups. We show that all exceptional characters in $B$ have the same Frobenius-Schur indicators. Moreover the common indicator can be computed, using the canonical character of $B$. We also investigate the Frobenius-Schur indicators of the non-exceptional characters in $B$.

For a finite group which has cyclic Sylow $p$-subgroups, we show that the number of irreducible characters with Frobenius-Schur indicator -1 is greater than or equal to the number of conjugacy classes of weakly real $p$-elements in $G$.
\end{abstract}

\section{INTRODUCTION AND PRELIMINARY RESULTS}

The Frobenius-Schur (F-S) indicator of an ordinary character $\chi$ of a finite group $G$ is

$$
\epsilon(\chi):=\frac{1}{|G|} \sum_{g \in G} \chi\left(g^{2}\right) .
$$

If $\chi$ is irreducible then $\epsilon(\chi)=0, \pm 1$. Moreover $\epsilon(\chi) \neq 0$ if and only if $\chi$ is real-valued.

R. Brauer showed how to partition the irreducible characters of $G$ into $p$-blocks, for each prime $p$. Each $p$-block has an associated defect group, which is a $p$-subgroup of $G$, unique up to $G$-conjugacy, which determines much of the structure of the block. If the defect group is trivial, the block contains a unique irreducible character. In the next most complicated case, E. Dade [D] determined the structure of a block which has a cyclic defect group and defined the Brauer tree of the block.

Recall that a $p$-block is said to be real if it contains the complex conjugates of its characters. We wish to determine the F-S indicators of the irreducible characters in a real $p$-block which has a cyclic defect group. In [M2, Theorem 1.6] we dealt with the case $p=2$; there are six possible indicator patterns, and the extended defect group of the block determines which occurs. In this paper we consider the case $p \neq 2$.

R. Gow showed [G, 5.1] that a real $p$-block has a real irreducible character, if $p=2$. This is false for $p \neq 2$, as was first noticed by H. Blau in the early 1980's, in response to a question posed by Gow. His example was for $p=5$ and $G=6 . S_{6}$ (Atlas notation). G. Navarro has recently found a solvable example with $p=3$ and $G=\operatorname{SmallGroup}(144,131)$ (GAP notation). We give examples for blocks with cyclic defect below.

Date: January 21, 2019.

2000 Mathematics Subject Classification. 20C20. 
Now let $B$ be a real $p$-block which has a cyclic defect group $D$. The inertial index of $B$ is a certain divisor $e$ of $p-1$. Dade showed that $B$ has $e$ irreducible Brauer characters and $e+\frac{|D|-1}{e}$ ordinary irreducible characters. The latter he divided into $\frac{|D|-1}{e}$ exceptional characters and $e$ non-exceptional characters.

Suppose that $\frac{|D|-1}{e}=1$ (which can only occur when $|D|=p$ ). Then the choice of exceptional character is arbitrary, and the convention in $[\mathrm{F}]$ is to regard $B$ as having no exceptional characters. However, we will see that in this event $B$ has real irreducible characters, all of which have the same F-S indicators. So our convention is to assume that $B$ has a real exceptional character.

The Brauer tree of $B$ is a planar graph which describes the decomposition matrix of $B$. There is one exceptional vertex, representating all the exceptional characters, and one vertex for each of the non-exceptional characters. Two vertices are connected by an edge if their characters share a modular constituent.

J. Green [Gr] showed that all real objects in the Brauer tree lie on a line segment, now called the real-stem of $B$. The exceptional vertex belongs to the real-stem (see Lemma 6 below). So it divides the real non-exceptional vertices into two, possibly empty, subsets. We find it convenient to refer to the corresponding real non-exceptional characters as being on the left or the right of the exceptional vertex. Here is our main theorem:

Theorem 1. Let $p$ be an odd prime and let $B$ be a real p-block which has a cyclic defect group. Then

(i) All exceptional characters in $B$ have the same F-S indicators.

(ii) On each side of the exceptional vertex, the real non-exceptional characters have the same F-S indicators.

(iii) If $B$ has a real exceptional character then all real irreducible characters in $B$ have the same F-S indicators.

(iv) Suppose that $B$ has no real exceptional characters, and that there are an odd number of non-exceptional vertices on each side of the exceptional vertex. Then the real non-exceptional characters have $F-S$ indicator +1 on one side of the exceptional vertex and -1 on the the other side.

Note that (i) is not a consequence of Galois conjugacy, as there are at least two Galois conjugacy classes of exceptional characters, when $|D|>p$.

In Proposition 15 we show that the F-S indicators of the exceptional characters in $B$ agree with those of the Brauer corresponding block in the normalizer of a defect group. In Theorem 16 we compute this common indicator using the 'canonical character' of $B$.

Next recall that an element of $G$ is said to be weakly real if it is conjugate to its inverse in $G$, but it is not inverted by any involution in $G$. Here is an application of Theorem 1 whose statement does not refer to blocks or to modular representation theory:

Theorem 2. Let $p$ be an odd prime and let $G$ be a finite group which has cyclic Sylow p-subgroups. Then the number of irreducible characters of $G$ with $F-S$ indicator -1 is greater than or equal to the number of conjugacy classes of weakly real p-elements in $G$. 
We use the notation and results of [NT] for group representation theory, and use [D] and [F, VII] for notation specific to blocks with cyclic defect. When referring to the character tables of a finite simple group we use the conventions of the ATLAS [A]. For other character tables, we use the notation of the computer algebra system GAP [GAP].

\section{EXAMPLES}

We begin with a number of examples which illustrate the possible patterns of F-S indicators in a block which has a cyclic defect group. Throughout $G$ is a finite group and $B$ is a real $p$-block of $G$ which has a cyclic defect group $D$. Also $N_{0}$ is the normalizer in $G$ of the unique order $p$ subgroup of $D$ and $B_{0}$ is the Brauer correspondent of $B$ in $N_{0}$.

Example 1: There are many blocks with cyclic defect group whose irreducible characters all have the same F-S indicators. For blocks with all indicators +1 , choose $n \geq 2$, a prime $p$ with $n / 2 \leq p \leq n$ and any $p$-block of the symmetric group $S_{n}$. There are numerous blocks with all indicators -1 among the faithful $p$-blocks of the double cover 2. $A_{n}$ of an alternating group, with $n / 2 \leq p \leq n$ e.g. the four faithful irreducible characters of 2. $A_{5}$ have $\mathrm{F}-\mathrm{S}$ indicator -1 and constitute a 5 -block with a cyclic defect group.

Example 2: If $e$ is odd then $B$ has a real non-exceptional character. Now it follows from [D, Part 2 of Theorem 1 \& Corollary 1.9] that $B$ has a Galois conjugacy class consisting of $\frac{p-1}{e}$ exceptional characters. So $B$ has a real exceptional character if $\frac{p-1}{e}$ is odd. Thus $B$ always has a real irreducible character if $p \equiv 3(\bmod 4)$.

When $e$ is even and $p \equiv 1(\bmod 4), B$ may have no real irreducible characters. For example SmallGroup $(80,29)=\left\langle a, b \mid a^{20}, a^{10}=b^{4}, a^{b}=a^{7}\right\rangle$ has such a block, for $p=5$. It consists of the four irreducible characters lying over the non-trivial irreducible character of $\left\langle a^{10}\right\rangle$. Here is its character table. The first two rows indicate the 2 and 5 parts of the class centralizers. The third row labels the classes by their element orders:

$\begin{array}{lrrrrrrrrrrrrrrrrr} & 2 & 4 & 4 & 3 & 3 & 4 & 4 & 2 & 3 & 3 & 3 & 3 & 2 & 2 & 2 \\ & 5 & 1 & 1 & . & 1 & . & . & 1 & . & . & . & . & 1 & 1 & 1 \\ & & 1 a & 2 a & 2 b & 4 a & 4 b & 4 c & 5 a & 8 a & 8 b & 8 c & 8 d & 10 a & 20 a & 20 b \\ X .9 & & 2 & -2 & . & . & 2 i & -2 i & 2 & . & . & . & & & & & \\ X .10 & 2 & -2 & . & . & -2 i & 2 i & 2 & . & . & . & . & -2 & . & . \\ X .13 & 4 & -4 & . & . & . & . & -1 & . & . & . & . & 1 & \sqrt{-5} & -\sqrt{-5} \\ X .14 & 4 & -4 & . & . & . & . & -1 & . & . & . & . & 1 & -\sqrt{-5} & \sqrt{-5}\end{array}$

Note that $\operatorname{SmallGroup}(80,29)$ has Sylow 2-subgroups isomorphic to $\operatorname{SmallGroup}(16,6)=$ $\left\langle s, t \mid s^{8}, t^{2}, s^{t}=s^{5}\right\rangle$. This 2-group is sometimes denoted $M_{4}(2)$.

Example 3: $B$ may have a real non-exceptional character but no real exceptional characters. For example SmallGroup $(60,7)=\left\langle a, b \mid a^{15}, b^{4}, a^{b}=a^{2}\right\rangle$ has such a block, for $p=5$. It consists of the four irreducible characters lying over a non-trivial irreducible 
character of $\left\langle a^{5}\right\rangle$. This is also an example of part (iv) of Theorem 1, the non-exceptional characters $X .5$ and $X .6$ have F-S indicators -1 and +1 , respectively. Here is the table of character values, with $\alpha=(1+\sqrt{-15}) / 2$ :

$\begin{array}{rrrrrrrrrr}2 & 2 & 2 & 1 & 2 & 2 & . & 1 & \cdot & . \\ 3 & 1 & 1 & 1 & \cdot & \cdot & 1 & 1 & 1 & 1 \\ 5 & 1 & \cdot & 1 & \cdot & \cdot & 1 & . & 1 & 1 \\ & 1 a & 2 a & 3 a & 4 a & 4 b & 5 a & 6 a & 15 a & 15 b\end{array}$

$\begin{array}{rrrrllrrrr}X .5 & 2 & -2 & -1 & . & . & 2 & 1 & -1 & -1 \\ X .6 & 2 & 2 & -1 & . & . & 2 & -1 & -1 & -1 \\ X .8 & 4 & . & -2 & . & . & -1 & . & \alpha & \bar{\alpha} \\ X .9 & 4 & . & -2 & . & . & -1 & . & \bar{\alpha} & \alpha\end{array}$

Example 4: There is no apparent relationship between the F-S indicators of the nonexceptional characters in $B$ and in $B_{0}$. For example, let $B$ be the 5 -block $2 . A_{8}$ with $\operatorname{Irr}(B)=\left\{\chi_{15}, \chi_{19}, \chi_{21}, \chi_{22}\right\}$. Then the two non-exceptional characters $\chi_{15}$ and $\chi_{19}$ have F-S indicator +1 and -1 , respectively. However $B_{0}$ is a real block which has no real irreducible characters.

The character table of $B$ can be found on p22 of The Atlas. Now $N_{0}$ is isomorphic to $\operatorname{SmallGroup}(120,7)=\left\langle a, b \mid a^{15}, b^{8}, a^{b}=a^{2}\right\rangle$. Here is the table of character values of its 5-block $B_{0}$. Again $\alpha=(1+\sqrt{-15}) / 2$. In order to save space, we have omitted 4 columns of zero values for the four classes of elements of order 8 :

$\begin{array}{rrrrrrrrrrrrrrr}2 & 3 & 3 & 2 & 3 & 3 & 1 & 2 & 1 & 2 & 2 & 1 & 1 & 1 & 1 \\ 3 & 1 & 1 & 1 & 1 & 1 & 1 & 1 & 1 & 1 & 1 & 1 & 1 & 1 & 1 \\ 5 & 1 & 1 & 1 & . & . & 1 & 1 & 1 & . & . & 1 & 1 & 1 & 1 \\ & 1 a & 2 a & 3 a & 4 a & 4 b & 5 a & 6 a & 10 a & 12 a & 12 b & 15 a & 15 b & 30 a & 30 b\end{array}$

$\begin{array}{lllllllllllllll}X .11 & 2 & -2 & -1 & 2 i & -2 i & 2 & 1 & -2 & -i & i & -1 & -1 & 1 & 1\end{array}$

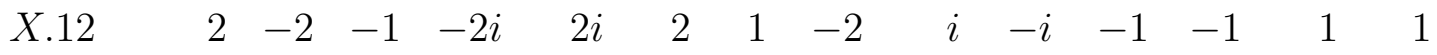

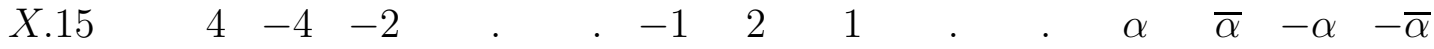

$\begin{array}{lllllllllllllllll}X .16 & 4 & -4 & -2 & & . & & . & -1 & 2 & 1 & & & & & & \end{array}$

We note that $B$ has 2 irreducible modules and 2 weights, in conformity with Alperin's weight conjecture [Al]. However the irreducible modules are self-dual and the weights are duals of each other. This shows that there is no obvious 'real' version of the weight conjecture for $p$-blocks, when $p \neq 2$.

Consider the inclusion of groups $N_{0}<\mathrm{PSL}_{2}(11)<M_{11}$, where $N_{0} \cong 11: 5$. The principal 11-blocks each have 5 non-exceptional characters. It is somewhat surprising that the number of real non-exceptional characters in these blocks is 1,5 and 3 , respectively. 
Example 5: Finally $B$ may have a real exceptional character but no real non-exceptional characters. For example let $B$ be the 5-block containing the four faithful irreducible characters of $\operatorname{SmallGroup}(20,1)=\left\langle a, b \mid a^{5}, b^{4}, a^{b}=a^{-1}\right\rangle$. The two exceptional characters have F-S indicators -1 , but neither of the two non-exceptional characters is real. Here is the character table of $B$, with $\beta=(-1+\sqrt{5}) / 2$ and $* \beta=(-1-\sqrt{5}) / 2$ :

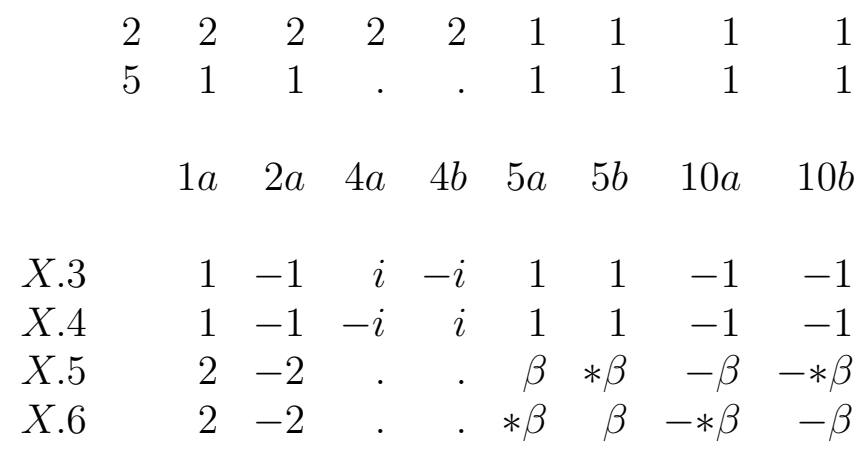

3. Miscellaneous Results

We need general results from representation theory, some of which are not so wellknown. So in this section $p$ is a prime and $B$ is a $p$-block of a finite group $G$.

Let $\chi$ be an irreducible character in $B$, let $x$ be a $p$-element of $G$ and let $y$ be a $p$-regular element of $\mathrm{C}_{G}(x)$. Then

$$
\chi(x y)=\sum_{\varphi} d_{\chi, \varphi}^{(x)} \varphi(y),
$$

where $\varphi$ ranges over the irreducible Brauer characters in blocks of $\mathrm{C}_{G}(x)$ which Brauer induce to $B$, and each $d_{\chi, \varphi}^{(x)}$ is an algebraic integer, called a generalized decomposition number; if $x=1, \varphi$ is an irreducible Brauer character in $B$ and $d_{\chi, \varphi}^{(x)}$ is simplified to $d_{\chi, \varphi}$. It is an integer called an ordinary decomposition number of $B$.

Brauer [B, Theorem (4A)] used his Second Main Theorem to prove the following remarkable 'local-to-global' formula for F-S indicators:

$$
\sum_{\chi} \epsilon(\chi) d_{\chi, \varphi}^{(x)}=\sum_{\psi} \epsilon(\psi) d_{\psi, \varphi}^{(x)}
$$

where $\chi$ ranges over the irreducible characters in $B$ and $\psi$ ranges over the irreducible characters in blocks of $\mathrm{C}_{G}(x)$ which Brauer induce to $B$. We have previously used this formula to determine the F-S indicators of the irreducible characters in 2-blocks with a cyclic, Klein-four or dihedral defect group.

Our next result relies on Clifford theory. However it was inspired by (and can be proved using) the notion of a weakly real 2-block, as introduced in [M1]. Suppose that $N$ is a normal subgroup of $G$ and $\phi \in \operatorname{Irr}(N)$, with stabilizer $G_{\phi}$ in $G$. If $G_{\phi} \subseteq H \subseteq G$, the Clifford correspondence is a bijection $\operatorname{Irr}(G \mid \phi) \leftrightarrow \operatorname{Irr}(H \mid \phi)$ such that $\chi \leftrightarrow \psi$ if and only if $\left\langle\chi \downarrow_{H}, \phi\right\rangle \neq 0$ or $\chi=\psi \uparrow G$. The stabilizer of $\{\phi, \bar{\phi}\}$ in $G$ is called the extended stabilizer of $\phi$, here denoted by $G_{\phi}^{*}$. So $\left|G_{\phi}^{*}: G_{\phi}\right| \leq 2$, with equality if and only if $\phi \neq \bar{\phi}$ but $\phi$ 
is $G$-conjugate to $\bar{\phi}$. If $G_{\phi}^{*} \subseteq H$ it is easy to see that $\chi$ is real if and only if $\psi$ is real. Moreover in this case $\epsilon(\chi)=\epsilon(\psi)$.

We need one other idea. Suppose that $T$ is a degree 2 extension of $G$. Then the Gow indicator [G, 2.1] of a character $\chi$ of $G$ with respect to $T$ is defined to be

$$
\epsilon_{T / G}(\chi):=\frac{1}{|G|} \sum_{t \in T \backslash G} \chi\left(t^{2}\right) .
$$

Clearly $\epsilon\left(\chi \uparrow^{T}\right)=\epsilon(\chi)+\epsilon_{T / G}(\chi)$. Just like the F-S indicator, $\epsilon_{T / G}(\chi)=0, \pm 1$, for each $\chi \in \operatorname{Irr}(G)$. Moreover $\epsilon_{T / G}(\chi) \neq 0$ if and only if $\chi$ is $T$-conjugate to $\bar{\chi}$.

Lemma 3. Let $N$ be a normal odd order subgroup of $G$ and let $\phi \in \operatorname{Irr}(N)$. Suppose that $G_{\phi}^{*}$ does not split over $G_{\phi}$. Then there exists $\chi \in \operatorname{Irr}(G \mid \phi)$ such that $\epsilon(\chi)=-1$.

Proof. We first show that there exists $\psi \in \operatorname{Irr}(G \mid \phi)$ such that $\epsilon(\psi)=+1$. For let $S$ be a Sylow 2-subgroup of $G$. As $\phi^{\uparrow} G$ vanishes on the 2-singular elements of $G$, we have $\left(\phi \uparrow^{G}\right) \downarrow_{S}=\frac{\phi(1)|G|}{|N||S|} \rho_{S}$, where $\rho_{S}$ is the regular character of $S$. Now $\frac{\phi(1)|G|}{|N||S|}$ is an odd integer. So $\left\langle\left(\phi \uparrow^{G}\right) \downarrow_{S}, 1_{S}\right\rangle$ is odd. Moreover $\phi^{\uparrow}{ }^{G}$ is a real character of $G$. So $\left\langle\left(\phi \uparrow^{G}\right), \psi\right\rangle=\langle(\phi \uparrow G), \bar{\chi}\rangle$, for each $\psi \in \operatorname{Irr}(G)$. Pairing each irreducible character of $G$ with its complex conjugate, we see that there exists a real-valued $\psi \in \operatorname{Irr}(G \mid \phi)$ such that $\left\langle\psi_{\downarrow_{S}}, 1_{S}\right\rangle$ is odd. Then $\epsilon(\psi)=\epsilon\left(1_{S}\right)=+1$.

Following the discussion before the lemma, we may assume that $G=G_{\phi}^{*}$. So $\left|G: G_{\phi}\right|=$ 2. Next suppose that $g \in G$ and $\phi \uparrow^{G_{\phi}}\left(g^{2}\right) \neq 0$. Write $g=x y=y x$, where $x$ is a 2-element and $y$ is a 2-regular element. Then $g^{2}=x^{2} y^{2}$. As $\phi \uparrow G_{\phi}$ vanishes off $N$, we have $x^{2}=1$ and $y^{2} \in N$. So $x \in G_{\phi}$, as $G_{\phi}$ contains all involutions in $G$. Moreover $y \in N$, as $y$ has odd order. Thus $g \in G_{\phi}$, whence

$$
\epsilon_{G / G_{\phi}}\left(\phi \uparrow^{G_{\phi}}\right)=\frac{1}{\left|G_{\phi}\right|} \sum_{g \in G \backslash G_{\phi}} \phi \uparrow^{G_{\phi}}\left(g^{2}\right)=0 .
$$

Now $\operatorname{Irr}\left(G_{\phi} \mid \phi\right)$ contains no real characters, as $\phi \neq \bar{\phi}$. So $\epsilon\left(\phi \uparrow^{G}\right)=\epsilon_{G / G_{\phi}}\left(\phi \uparrow G_{\phi}\right)+$ $\epsilon\left(\phi \uparrow^{G_{\phi}}\right)=0$. Equivalently

$$
\sum_{\chi \in \operatorname{Irr}(G)}\left\langle\phi \uparrow^{G}, \chi\right\rangle \epsilon(\chi)=0
$$

Together with the fact that $\left\langle\phi \uparrow^{G}, \psi\right\rangle \epsilon(\psi)>0$, this implies that $\langle\phi \uparrow G, \chi\rangle \epsilon(\chi)<0$, for some $\chi \in \operatorname{Irr}(G)$. Thus $\chi \in \operatorname{Irr}(G \mid \phi)$ and $\epsilon(\chi)=-1$, which completes the proof.

It is well-known that each $G$-invariant irreducible character of a normal subgroup of $G$ extends to $G$, when the quotient group is cyclic.

Lemma 4. Suppose that $N$ is a normal subgroup of $G$ such that $G / N$ is cyclic and of even order. Let $\varphi \in \operatorname{Irr}(N)$ be real and $G$-invariant. Then $\varphi$ has a real extension to $G$ if and only if $\varphi$ has a real extension to $T$, where $N \subset T \subseteq G$ and $T / N$ has order 2 . 
Proof. The 'only if' part is obvious. So assume that $\varphi$ has a real extension to $T$. Then both extensions of $\varphi$ to $T$ are real. Let $\omega$ be a generator of the abelian group $\operatorname{Irr}(G / N)$ and let $\chi$ be any extension of $\varphi$ to $G$. Then $\omega^{i} \chi, i \geq 0$ give all extensions of $\varphi$ to $G$. Here $\omega^{i}=\omega^{j}$ if and only if $i \equiv j(\bmod |G / N|)$.

As $\bar{\chi}$ lies over $\varphi$, we have $\bar{\chi}=\omega^{i} \chi$, for some $i \geq 0$. Now $\chi \downarrow_{T}$ is an extension of $\varphi$ to $T$ and $\bar{\chi} \downarrow_{T}=\left(\omega^{i} \downarrow_{T}\right)\left(\chi \downarrow_{T}\right)$. As $\chi_{\downarrow_{T}}$ is real, it follows that $\omega^{i} \downarrow_{T}$ is trivial. So $T \subseteq \operatorname{ker}\left(\omega^{i}\right)$, whence $i \equiv 2 j(\bmod |G / N|)$, for some $j \geq 0$. Now $\overline{\omega^{j} \chi}=\omega^{i-j} \chi=\omega^{j} \chi$. So $\omega^{j} \chi$ is a real extension of $\varphi$ to $G$.

Notice that in this context $\varphi$ has a real extension to $T$ if and only if $\epsilon(\varphi)=\epsilon_{T / N}(\varphi)$. When $G / N$ has even order, but is not cyclic, and $\varphi$ is a real irreducible character of $N$ which extends to $G$, it is not clear whether there is a sensible sufficient criteria for $\varphi$ to have a real extension to $G$.

Finally we need the following consequence of the first orthogonality relation:

Lemma 5. Let $W \subseteq X \subseteq Y$ be finite abelian groups. Then for $\lambda \in \operatorname{Irr}(Y)$ we have

$$
\sum_{x \in X \backslash W} \lambda(x)=\left\{\begin{aligned}
|X|-|W|, & \text { if } X \subseteq \operatorname{ker}(\lambda) . \\
-|W|, & \text { if } W \subseteq \operatorname{ker}(\lambda) \text { but } X \nsubseteq \operatorname{ker}(\lambda) . \\
0, & \text { if } W \nsubseteq \operatorname{ker}(\lambda) .
\end{aligned}\right.
$$

\section{The Brauer tree And its REAL-STEM}

From now on $G$ is a finite group, $p$ is an odd prime and $B$ is a real $p$-block of $G$ which has a cyclic defect group. To avoid trivialities we assume that the defect group is non-trivial.

Dade asserts [D, Theorem 1, Part 2] that each decomposition number in $B$ is either 0 or 1 . The Brauer tree of $B$ is a planar graph with edges labelled by the irreducible Brauer character in $B$ and with vertices labelled by the irreducible characters in $B$ (the exceptional characters in $B$ label a single 'exceptional' vertex). The edge labelled by an irreducible Brauer character $\theta$ meets the vertex labelled by an irreducible character $\chi$ if and only if the decomposition number $d_{\chi, \theta}$ is not 0 .

When $B$ is real, complex conjugation acts on the Brauer tree of $B$, and in particular fixes the exceptional vertex. However, as we have seen in Examples 2,3 and 4 above, $B$ may have no real exceptional characters. So we restate [F, VII,9.2] in the following more precise fashion:

Lemma 6. The subgraph of the Brauer tree of B consisting of the exceptional vertex and those vertices and edges which correspond to real characters and Brauer characters is a straight line segment.

Feit calls this line segment the real-stem of $B$. An easy consequence is:

Corollary 7. The number of real non-exceptional characters in $B$ equals the number of real irreducible Brauer characters in B. 
Proof. Suppose that $B$ has $r$ real irreducible Brauer characters. Then the real-stem of the Brauer tree has $r$ edges and $r+1$ vertices. One of these is the exceptional vertex. So $B$ has $r$ real non-exceptional characters.

Let $\theta$ be a real irreducible $p$-Brauer character of a finite group $G$. As $p$ is odd, the $G$-representation space of $\theta$ affords a non-degenerate $G$-invariant bilinear form which is either symmetric or skew-symmetric. Given the symmetry groups of such forms, we refer to $\theta$ as being of orthogonal or symplectic type. Thompson and Willems [W, 2.8] proved that there is a real irreducible character $\chi$ of $G$ such that $d_{\chi, \theta}$ is odd. Moreover $\theta$ has orthogonal type if $\epsilon(\chi)=+1$ or symplectic type if $\epsilon(\chi)=-1$. This implies that $\epsilon(\psi)=\epsilon(\chi)$, for all real irreducible characters $\psi$ such that $d_{\psi, \theta}$ is odd.

Proof of part (ii) of Theorem 1. Let $X$ and $Y$ be real non-exceptional characters which lie on the same side of the exceptional vertex in the real-stem of $B$. Then by Lemma 6] there is a sequence $X=X_{0}, X_{1}, \ldots, X_{n}=Y$ of real non-exceptional characters and a sequence $\theta_{1}, \ldots, \theta_{n}$ of real irreducible Brauer characters such that $d_{X_{i-1}, \theta_{i}}=1=d_{X_{i}, \theta_{i}}$, for $i=1, \ldots, n$. The Thompson-Willems result implies that $\epsilon\left(X_{i-1}\right)=\epsilon\left(X_{i}\right)$, for $i=1, \ldots, n$. So $\epsilon(X)=\epsilon(Y)$. This gives part (ii) of Theorem 1,

A similar argument gives the following weak form of parts (i) and (iii) of Theorem 1;

Lemma 8. If $B$ has a real exceptional character and a real non-exceptional character, then all real irreducible characters in $B$ have the same $F$-S indicators.

Notice that if $B$ is the principal $p$-block of a group with a cyclic Sylow $p$-subgroup, and $B$ has an irreducible character with F-S indicator -1 (e.g. the principal 7-block of $\mathrm{U}(3,3))$ then the lemma implies that $B$ has no real exceptional characters.

\section{THE EXCEPTIONAL CHARACTERS}

We outline some results from [D] using the language of subpairs. See [NT, Chapter 5.9] for a full description of the theory. We then prove results about the local blocks in $B$, in Proposition 10, and the exceptional characters in $B$, in Proposition 11. This allows us to prove parts (i), (iii) and (iv) of Theorem 1.

Recall that $B$ is a $p$-block with a non-trivial cyclic defect group $D$. Write $|D|=p^{a}$, where $a>0$, and let $1 \subset D_{a-1} \subset D_{a-2} \subset \cdots \subset D_{1} \subset D_{0}=D$ be the complete list of subgroups of $D$. So $\left[D: D_{i}\right]=p^{i}$, for $i=0, \ldots, a-1$. Set $C_{i}=C_{G}\left(D_{i}\right)$ and $N_{i}=N_{G}\left(D_{i}\right)$. So $C_{0} \subseteq C_{1} \subseteq \ldots \subseteq C_{a-1}$, and $N_{0} \subseteq N_{1} \subseteq \ldots \subseteq N_{a-1}$.

As $p$ is odd, $\operatorname{Aut}\left(D_{i}\right)$ is a cyclic group of order $p^{a-i-1}(p-1)$. So $N_{i} / C_{i}$ is a cyclic group whose order divides $p^{a-i-1}(p-1)$. Moroever the centralizer of $D_{i}$ in $\operatorname{Aut}(D)$ has order $p^{i}$. So $C_{i} \cap N_{0} / C_{0}$ is a cyclic $p$-group. We note that the unique involution in $\operatorname{Aut}(D)$ inverts every element of $D$.

Fix a Sylow $B$-subpair $\left(D, b_{0}\right)$. So $b_{0}$ is a $p$-block of $C_{0}$ such that $b_{0}^{G}=B$ and the pair $\left(D, b_{0}\right)$ is uniquely determined up to $G$-conjugacy. Set $b_{i}:=b_{0}^{C_{i}}$, for $i=1, \ldots, a-1$. Then 
by [NT, 5.9.3] the lattice of $B$-subpairs contained in $\left(D, b_{0}\right)$ is

$$
(1, B) \subset\left(D_{a-1}, b_{a-1}\right) \subset \cdots \subset\left(D_{1}, b_{1}\right) \subset\left(D, b_{0}\right) .
$$

Set $E:=\mathrm{N}\left(D, b_{0}\right)$, the stabilizer of $b_{0}$ in $N_{0}$. Then $e:=\left|E: C_{0}\right|$ is called the inertial index of $B$. Now $p \nmid e$, by Brauer's extended first main theorem. So $e \mid(p-1)$. Let $x \in E$. Then $D_{i}^{x}=D_{i}$. As $\left(D_{i}, b_{i}\right),\left(D_{i}, b_{i}^{x}\right) \subseteq\left(D, b_{0}\right)$, it follows from (2) that $b_{i}^{x}=b_{i}$. So $E C_{i} \subseteq \mathrm{N}\left(D_{i}, b_{i}\right)$. Conversely let $n \in \mathrm{N}\left(D_{i}, b_{i}\right)$. As $\left(D, b_{0}\right)$ and $\left(D, b_{0}\right)^{n}$ are Sylow $b_{i}$-subpairs (in the group $C_{i}$ ), there is $c \in C_{i}$ such that $n c_{i} \in E$. This shows that $\mathrm{N}\left(D_{i}, b_{i}\right) \subseteq E C_{i}$. This recovers Dade's observation that $\mathrm{N}\left(D_{i}, b_{i}\right)=E C_{i}$.

Now $E \cap C_{i} / C_{0}$ is a subgroup of $C_{i} \cap N_{0} / C_{0}$ and a quotient of the group $E / C_{0}$. As $C_{i} \cap N_{0} / C_{0}$ is a $p$-group and $E / C_{0}$ has $p^{\prime}$-order, we deduce that $E \cap C_{i}=C_{0}$. It follows from this $E C_{i} / C_{i} \cong E / C_{0}$, and in particular $\left|E C_{i}: C_{i}\right|=e$.

By [D, Theorem 1, Part 1] $B$ has $e$ irreducible Brauer characters, listed as $\chi_{1}, \ldots, \chi_{e}$. Each $b_{i}$ has inertial index 1. So $b_{i}$ has a unique irreducible Brauer character, denoted $\varphi_{i}$.

From the above discussion there are $\left|N_{i}: E C_{i}\right|=\frac{\left|N_{i}: C_{i}\right|}{e}$ distinct blocks of $C_{i}$ which induce to $B$, namely $b_{i}^{\tau}$ as $\tau$ ranges over $N_{i} / E C_{i}$. Also there are $\frac{p^{a-i}-p^{a-i-1}}{\left|N_{i}: C_{i}\right|}$ conjugacy classes of $G$ which contain a generator of $D_{i}$. So $B$ has $\frac{p^{a-i}-p^{a-i-1}}{e}$ subsections $(x, b)$, with $D_{i}=\langle x\rangle$. A consequence of Brauer's second main theorem [NT, 5.4.13(ii)] is that the number of irreducible characters in a block equals the number of columns in the block.

Lemma 9. A complete set of columns of $B$ is

$$
\left(1, \chi_{1}\right), \ldots,\left(1, \chi_{e}\right), \quad\left(x_{i}^{\sigma_{i}}, \varphi_{i}^{n_{i}}\right), \quad i=0, \ldots, a-1 .
$$

Here $x_{i}$ is a fixed generator of $D_{i}, \sigma_{i}$ ranges over a set of representatives for the cosets of the image of $N_{i} / C_{i}$ in $\operatorname{Aut}\left(D_{a-i}\right)$ and $n_{i}$ ranges over a set of representatives for the cosets of $E C_{i}$ in $N_{i}$. In particular $k(B)=e+\frac{p^{a}-1}{e}$.

Let $\Lambda$ be a set of representatives for the $\frac{p^{a}-1}{e}$ orbits of $E$ on $\operatorname{Irr}(D)^{\times}$. Then

$$
\operatorname{Irr}(B)=\left\{X_{1}, \ldots, X_{e}\right\} \bigcup\left\{X_{\lambda} \mid \lambda \in \Lambda\right\} .
$$

Also set $X_{0}:=\sum_{\lambda \in \Lambda} X_{\lambda}$. Dade refers to the $X_{\lambda}$ as the exceptional characters of $B$.

Notice that as $\ell\left(b_{i}\right)=1, b_{i}$ is real if and only if $\varphi_{i}$ is real. The next two propositions are relatively elementary.

Proposition 10. All the blocks $b_{0}, b_{1}, \ldots, b_{a-1}$ are real or none of them are real.

Proof. We have $\left(b_{i}^{o}\right)^{G}=B^{o}=B$. So $\left(D, b_{0}\right)$ and $\left(D, b_{o}^{o}\right)$ are Sylow $B$-subpairs, and there is $n \in \mathrm{N}_{0}$ such that $b_{0}^{o}=b_{0}^{n}$.

Suppose that $b_{j}$ is real, for some $j=0, \ldots, a-1$. As $\left(D_{j}, b_{j}^{n}\right),\left(D_{j}, b_{j}^{o}\right) \subset\left(D_{0}, b_{0}^{o}\right)$, it follows from (2) that $b_{j}^{n}=b_{j}^{o}=b_{j}$. So $n \in \mathrm{N}\left(D_{j}, b_{j}\right)=E C_{j}$. Write $n=e c$, where $e \in E$ and $c \in C_{j}$. Then $c=e^{-1} n \in C_{j} \cap N_{0}$ and $b_{0}^{c}=b_{0}^{n}=b_{0}^{o}$. So $c^{2} \in C_{j} \cap E=C_{0}$. But $C_{j} \cap N_{0} / C_{0}$ has odd order, as it is a $p$-group. So $c \in C_{0}$, which shows that $n \in E$. As $b_{0}^{n}=b_{0}$, it follows that $b_{0}$ is real. 
Now let $i=0, \ldots, a-1$. Then $\left(D_{i}, b_{i}\right),\left(D_{i}, b_{i}^{o}\right) \subset\left(D_{0}, b_{0}\right)=\left(D_{0}, b_{0}^{o}\right)$. So $b_{i}=b_{i}^{o}$, for $i=0, \ldots, a-1$, using (2). This shows that all $b_{0}, \ldots, b_{a-1}$ are real.

We showed in [M3, 1.1] that the number of real irreducible characters in a block equals the number of real columns in the block. Here $(x, \varphi)$ is real if $x^{g}=x^{-1}$ and $\varphi^{g}=\bar{\varphi}$, for some $g \in G$.

Let $i=0, \ldots, a-1$. As $b_{i}$ has inertial index 1, it has $|D|$ irreducible characters. Modifying [D, p26] we use the notation

$$
\operatorname{Irr}\left(b_{i}\right)=\left\{X_{i, \lambda}^{\prime} \mid \lambda \in \operatorname{Irr}(D)\right\}
$$

Here $X_{i, 1}^{\prime}$ is the unique non-exceptional character in $b_{i}$, and all characters $X_{i, \lambda}^{\prime}$ with $\lambda \neq 1$ are exceptional. Suppose that $b_{i}$ is real. The columns of $b_{i}$ are $\left(d, \varphi_{i}\right)$, for $d \in D$. As $C_{i}$ acts trivially on the columns, the only real column is $\left(1, \varphi_{i}\right)$. So $X_{i, 1}^{\prime}$ is the only real irreducible character in $b_{i}$.

We will refine the next result in part (i) of Theorem 1:

Proposition 11. All exceptional characters in $B$ are real or none are real.

Proof. It follows from Corollary 7 and Lemma 9 that the number of real exceptional characters in $B$ equals the number of real columns $(x, \varphi)$ with $x \in D^{\times}$and $\varphi \in \operatorname{IBr}\left(\mathrm{C}_{G}(x)\right)$.

Suppose that $B$ has a real exceptional character, and let $(x, \varphi)$ be a real column of $B$, with $x \in D^{\times}$. Then $\langle x\rangle=D_{i}$, for some $i=0, \ldots, a-1$. As $N_{i} / C_{i}$ is abelian, the columns $\left(x^{\prime}, \varphi_{i}^{n_{i}}\right)$ are real, for all generators $x^{\prime}$ of $D_{i}$ and all $n_{i} \in N_{i}$. In particular $\left(x_{i}, \varphi_{i}\right)$ is a real column. Choose $n \in N_{i}$ such that $x_{i}^{n}=x_{i}^{-1}$ and $\varphi_{i}^{n}=\bar{\varphi}_{i}$. We may suppose that $n^{2} \in C_{i}$.

Suppose first that $b_{i}$ is real. As $\varphi_{i}=\bar{\varphi}_{i}, n$ fixes $\varphi_{i}$ and inverts $D_{i}$. So $n C_{i}$ is an involution in $E C_{i} / C_{i}$. As $E C_{i} / C_{i} \cong E / C_{0}$, we may assume without loss that $n C_{0}$ is an involution in $E / C_{0}$. Now all the blocks $b_{0}, \ldots, b_{a-1}$ are real. Hence all $\varphi_{0}, \ldots, \varphi_{a-1}$ are real. As $n$ inverts $D_{j}$ and fixes $\varphi_{j}$, all columns $\left(x_{j}, \varphi_{j}\right)$ are real. Thus all columns $(x, \varphi)$, with $x \in D^{\times}$, are real. So all exceptional characters in $B$ are real in this case.

Conversely, suppose that $b_{i}$ is not real. As $n C_{i}$ is the unique involution in $N_{i} / C_{i}$, but $n \notin E C_{i}$, it follows that $\left|E C_{i}: C_{i}\right|=e$ is odd. Now $\left(D, b_{0}\right)$ and $\left(D, b_{0}^{o}\right)$ are Sylow $B$ subpairs, but $b_{0} \neq b_{0}^{o}$. So there is $m \in N_{0} \backslash E$ such that $b_{0}^{m}=b_{0}^{o}$. As $m^{2} \in E$ and $\left|E: C_{0}\right|$ is odd, we may choose $m$ so that $m^{2} \in C_{0}$. Then $m C_{0}$ is the unique involution in $N_{0} / C_{0}$. In particular $m$ inverts every element of $D$. Let $j=0, \ldots, a-1$. Then $\left(D_{j}, b_{j}^{m}\right)$ and $\left(D_{j}, b_{j}^{o}\right)$ are $B$-subpairs contained in $\left(D, b_{0}^{o}\right)$. So $b_{j}^{m}=b_{j}^{o}$ and thus $\left(d_{j}, \varphi_{j}\right)^{m}=\left(d_{j}^{-1}, \bar{\varphi}_{j}\right)$. It follows that all exceptional characters in $B$ are real in this case also.

Examination of the proof shows that:

Corollary 12. All exceptional characters in $B$ are real if and only if $b_{0}$ is real and $e$ is even, or $b_{0}$ is not real and $e$ is odd.

We need some additional notation. Set $\Lambda_{u}:=\left\{\lambda \in \Lambda \mid \operatorname{ker}(\lambda)=D_{u}\right\}$, for $u=1, \ldots, a$. So $\left|\Lambda_{u}\right|=\frac{p^{u}-p^{u-1}}{e}$. Now choose $\lambda \in \Lambda_{u}$ and set

$$
\epsilon_{u}:=\epsilon\left(X_{\lambda}\right) .
$$


Note that $X_{\lambda}$ and $X_{\mu}$ are Galois conjugates, for all $\lambda, \mu \in \Lambda_{u}$ (this follows from [D, part 2 of Theorem 1 and Corollary 1.9]). So $\epsilon_{u}$ does not depend on $\lambda$.

Recall our notation (4) for the irreducible characters $X_{i, \lambda}^{\prime}$ in $b_{i}$. As already noted, $X_{i, 1}^{\prime}$ is the only possible real irreducible character in $b_{i}$. We set

$$
\nu_{i}:=\epsilon\left(X_{i, 1}^{\prime}\right), \quad \text { for } i=0, \ldots, a-1 .
$$

Now let $i=0, \ldots, a-1$ and choose $x \in D_{i}-D_{i+1}$ and $\rho \in N_{i}$. According to [D, Theorem 1, Part 3] there are signs $\varepsilon_{0}^{\prime}, \varepsilon_{0}, \varepsilon_{1} \ldots, \varepsilon_{e}$ and $\gamma_{i}$ such that

$$
\begin{array}{llrl}
d_{X_{\lambda}, \varphi_{i}^{\rho}}^{(x)} & =\varepsilon_{0} \gamma_{i} \sum_{\tau \in E C_{i} / C_{i}} \lambda\left({ }^{\rho \tau} x\right), & d_{X_{j}, \varphi_{i}^{\rho}}^{(x)} & =\varepsilon_{j} \gamma_{i}, \quad \text { for } j=1, \ldots, e \\
d_{X_{i, \lambda}^{\prime}, \varphi_{i}^{\rho}}^{(x)} & =\varepsilon_{0}^{\prime} \gamma_{i} \lambda\left({ }^{\rho} x\right), & d_{X_{i, 1}^{\prime}, \varphi_{i}^{\rho}}^{(x)} & =1
\end{array}
$$

Here $E C_{i} / C_{i}$ is a set of representatives for the cosets of $C_{i}$ in $E C_{i}$. Note that Feit uses the notation $\delta_{0}=-\varepsilon_{0}$ and $\delta_{j}=\varepsilon_{j}$, for $j=1, \ldots, e$. Now let $i=0, \ldots, a-1$ and $x \in D_{i}-D_{i+1}$. Then it follows from [D, Corollary 1.9] that $X_{j}(x)=\left|N_{i}: E C_{i}\right| \varphi_{i}(1) \delta_{j} \gamma_{i}$. So $\delta_{j} \gamma_{i}$ is the sign of the integer $X_{j}(x)$.

There is a nice relationship between the signs $\varepsilon_{0}, \varepsilon_{1} \ldots, \varepsilon_{e}$ and the Brauer tree of $B$. Suppose that $j$ and $k$ are adjacent vertices in the Brauer tree. Then $X_{j}+X_{k}$ is a principal indecomposable character of $G$. So it vanishes on $D^{\times}$, and hence $\delta_{j}+\delta_{k}=0$ (see [F, V11, Section 9]). So suppose that there are $d_{j}$ edges between the vertex $j$ and the exceptional vertex 0 in the Brauer tree. Then $\delta_{j}=(-1)^{d_{j}} \delta_{0}$. So $\varepsilon_{j}=(-1)^{d_{j}-1} \varepsilon_{0}$, for $j=1, \ldots, e$.

We now prove part (i) of our main theorem. But note that this proof does not depend on Propositions 10 and [1]:

Proof of part (i) of Theorem 1. Applying (1), with $\rho \in N_{i}$ and $x \in D_{i}-D_{i+1}$, we get

$$
\sum_{j=1}^{e} \epsilon\left(X_{j}\right) \varepsilon_{j} \gamma_{i}+\sum_{\lambda \in \Lambda} \epsilon\left(X_{\lambda}\right) \varepsilon_{0} \gamma_{i} \sum_{\tau \in E C_{i} / C_{i}} \lambda\left({ }^{\rho \tau} x\right)=\nu_{i}
$$

Now set $\sigma:=\varepsilon_{0} \sum_{j=1}^{e} \epsilon\left(X_{j}\right) \varepsilon_{j}$. So $\sigma$ is independent of $i, \rho$ and $x$. Then the above equality transforms to

$$
\sum_{u=1}^{a} \epsilon_{u} \sum_{\lambda \in \Lambda_{u}} \sum_{\tau \in E C_{i}} \lambda\left({ }^{\rho \tau} x\right)=\varepsilon_{0} \gamma_{i} \nu_{i}-\sigma
$$

where the right hand side is independent of $\rho$ and $x$. Let $\rho$ range over a set of representatives for the $\frac{\left|N_{i}: C_{i}\right|}{e}$ cosets of $E C_{i}$ in $N_{i}$ and let $x$ range over a set of representatives for the $\frac{p^{a-i}-p^{a-i-1}}{\left|N_{i}: C_{i}\right|}$ orbits of $N_{i}$ on the generators of $D_{i}$. Then ${ }^{\rho \tau} x$ will range over all generators of $D_{i}$. Summing the resulting equalities gives

$$
\sum_{u=1}^{a} \epsilon_{u} \sum_{\lambda \in \Lambda_{u}} \sum_{x \in D_{i}-D_{i+1}} \lambda(x)=\left(\frac{p^{a-i}-p^{a-i-1}}{e}\right)\left(\varepsilon_{0} \gamma_{i} \nu_{i}-\sigma\right) .
$$


We use $\left|\Lambda_{u}\right|=\frac{p^{u}-p^{u-1}}{e}$ and Lemma 5 to transform this equality to

$$
\left(p^{a-i}-p^{a-i-1}\right) \sum_{u=1}^{i} \frac{p^{u}-p^{u-1}}{e} \epsilon_{u}-p^{a-i-1} \frac{p^{i+1}-p^{i}}{e} \epsilon_{i+1}=\frac{p^{a-i}-p^{a-i-1}}{e}\left(\varepsilon_{0} \gamma_{i} \nu_{i}-\sigma\right) .
$$

After cancelling the factor $\frac{p^{a-i-1}(p-1)}{e}$, we get

$$
\sum_{u=1}^{i}\left(p^{u}-p^{u-1}\right) \epsilon_{u}-p^{i} \epsilon_{i+1}=\varepsilon_{0} \gamma_{i} \nu_{i}-\sigma
$$

Here $\sum_{u=1}^{0}\left(p^{u}-p^{u-1}\right) \epsilon_{u}$ is taken to be 0 , when $i=0$. We write down the equalities (5) for $i=0,1,2, \ldots$ in turn:

$$
\begin{aligned}
-\epsilon_{1} & =\varepsilon_{0} \gamma_{0} \nu_{0}-\sigma \\
(p-1) \epsilon_{1}-p \epsilon_{2} & =\varepsilon_{0} \gamma_{1} \nu_{1}-\sigma \\
(p-1) \epsilon_{1}+\left(p^{2}-p\right) \epsilon_{2}-p^{2} \epsilon_{3} & =\varepsilon_{0} \gamma_{2} \nu_{2}-\sigma \\
(p-1) \epsilon_{1}+\left(p^{2}-p\right) \epsilon_{2}+\left(p^{3}-p^{2}\right) \epsilon_{3}-p^{3} \epsilon_{4} & =\varepsilon_{0} \gamma_{3} \nu_{3}-\sigma \\
\vdots & \\
(p-1) \epsilon_{1}+\left(p^{2}-p\right) \epsilon_{2}+\cdots+\left(p^{a-1}-p^{a-2}\right) \epsilon_{a-1}-p^{a-1} \epsilon_{a} & =\varepsilon_{0} \gamma_{a-1} \nu_{a-1}-\sigma
\end{aligned}
$$

Subtract the first equality from the second to get

$$
p\left(\epsilon_{1}-\epsilon_{2}\right)=\varepsilon_{0}\left(\gamma_{1} \nu_{1}-\gamma_{0} \nu_{0}\right) .
$$

The left hand side equals $-p, 0$ or $p$ and the right hand equals $-2,0$ or 2 . As $p$ is odd, the common value is 0 . So $\epsilon_{2}=\epsilon_{1}$ and $\gamma_{1} \nu_{1}=\gamma_{0} \nu_{0}$. Substitute these values back into all equations in (6). Now subtract the first from the third equality to get

$$
p^{2}\left(\epsilon_{1}-\epsilon_{3}\right)=\varepsilon_{0}\left(\gamma_{2} \nu_{2}-\gamma_{0} \nu_{0}\right) .
$$

Once again both sides are 0 . So $\gamma_{2} \nu_{2}=\gamma_{0} \nu_{0}$ and $\epsilon_{3}=\epsilon_{1}$. Proceeding in this way, we get

$$
\epsilon_{1}=\epsilon_{2}=\cdots=\epsilon_{a}, \quad \gamma_{0} \nu_{0}=\gamma_{1} \nu_{1}=\cdots=\gamma_{a-1} \nu_{a-1}
$$

Following the above proof, and the discussion before the proof, we obtain:

Corollary 13. Suppose that $b_{0}$ is real and let $D=\langle x\rangle$. Then for each $i=0, \ldots, a-1$ and $j=0, \ldots, e$, the integer $X_{j}\left(x^{p^{i}}\right) X_{j}(x)$ has sign $\epsilon\left(X_{i, 1}^{\prime}\right) \epsilon\left(X_{0,1}^{\prime}\right)$.

There is no apparent relationship between the F-S indicators $\nu_{0}, \ldots, \nu_{a-1}$ :

Example: The 2-nilpotent group $G=\left\langle a, b, c \mid a^{4}, a^{2}=b^{2}, a^{b}=a^{-1}, c^{9}, a^{c}=b, b^{c}=a b\right\rangle$ has isomorphism type 3 . SL $(2,3)$. Set $D=\langle c\rangle$. Then $D$ is cyclic of order 9 , with $C_{0}=$ $D \times\left\langle a^{2}\right\rangle$ and $C_{1}=G$. Let $\theta$ be the non-trivial irreducible character of $C_{0} / D$, and let $b_{0}$ be the 3 -block of $C_{0}$ which contains $\theta$. Then $\theta=X_{0,1}^{\prime}$ is the unique non-exceptional character 
in $b_{0}$. So $\nu_{0}=\epsilon\left(X_{0,1}^{\prime}\right)=+1$. Set $b_{1}=b_{0}^{G}$. Then $b_{1}$ also has a unique non-exceptional character $X_{1,1}^{\prime}$. But now $\nu_{1}=\epsilon\left(X_{1,1}^{\prime}\right)=-1$, as $X_{1,1}^{\prime}$ restricts to the non-linear irreducible character of $\langle a, b\rangle \cong Q_{8}$.

This example arises from the fact that the Glauberman correspondence [NT, 5.12] does not preserve the F-S indicators of characters.

proof of part (iii) of Theorem 1 . This is an immediate consequence of Lemma 8 and part (i) of Theorem 1 .

Consider the real-stem of $B$ as a horizontal line segment with $s$ vertices and $s-1$ edges, where $s \geq 1$. We label the vertices using an interval $[-\ell, \ldots,-2,-1,0,1,2, \ldots, r]$ so that 0 labels the exceptional vertex. Thus $s=r+\ell+1$, and there are $\ell$ real non-exceptional characters on the left of the exceptional vertex, and $r$ on the right (the choice of left and right is unimportant).

As above, $X_{0}$ is the sum of the exceptional characters in $B$. Now we relabel the nonexceptional characters in $B$ so that $X_{i}$ is the real non-exceptional character corresponding to vertex $i$, for $i=-\ell, \ldots, r$ and $i \neq 0$. In view of parts (i) and (ii) of Theorem 1 there are signs $\epsilon_{ \pm}$such that

$$
\epsilon\left(X_{i}\right)= \begin{cases}\epsilon_{-}, & \text {for } i=-\ell, \ldots,-1 . \\ \epsilon_{0}, & \text { for } i=0 . \\ \epsilon_{+}, & \text {for } i=1, \ldots, r .\end{cases}
$$

Next let $\sigma$ be a generator of $D$. It follows from [D, Corollary 1.9] that $X_{0}(\sigma)=$ $-\varepsilon_{0} \gamma_{0}\left|N_{0}: E\right| \varphi_{0}(1)$. So $X_{i}(\sigma)=(-1)^{i} X_{0}(\sigma)$, as $X_{i}+X_{i+1}$ is a projective character of $G$, for $i=-\ell, \ldots, r-1$ (see [F, VII,2.19(ii)]).

Recall from Section (5) that there are $\left|N_{0}: E\right|$ blocks of $C_{0}$ which induce to $B$; these are the blocks $b_{0}^{\tau}$, where $\tau$ ranges over $N_{0} / E$. We note also that $X_{0,1}^{\prime}\left({ }^{\tau} \sigma\right)=\varphi_{0}(1)$. Now $[\mathrm{B}$, Theorem(4B)] is an immediate consequence of [B, Theorem(4A)]. In our context, this states that

$$
\sum_{i=-\ell}^{r} \epsilon\left(X_{i}\right) X_{i}(\sigma)=\left|N_{0}: E\right| \epsilon\left(X_{0,1}^{\prime}\right) X_{0,1}^{\prime}(\sigma) .
$$

In view of the previous paragraph this simplifies to

$$
\sum_{i=1}^{\ell}(-1)^{i} \epsilon_{-}+\epsilon_{0}+\sum_{i=1}^{r}(-1)^{i} \epsilon_{+}=-\varepsilon_{0} \gamma_{0} \nu_{0} .
$$

We consider a number of cases.

Suppose first that $\epsilon_{0} \neq 0$. Then $\epsilon_{-}=\epsilon_{0}=\epsilon_{+}$, by part (iii) of Theorem 11. So (7) becomes

$$
-\varepsilon_{0} \gamma_{0} \nu_{0} \epsilon_{0}=\left\{\begin{array}{cl}
(-1)^{\ell}, & \text { if } s \text { is odd. } \\
0, & \text { if } s \text { is even. }
\end{array}\right.
$$

In particular $b_{0}$ is not real if $s$ is even. As $e$ is odd when $s$ is even, this already follows from Corollary 12 . 
Suppose then that $\epsilon_{0}=0$. Now (7) evaluates as

$$
-\varepsilon_{0} \gamma_{0} \nu_{0}=\left\{\begin{array}{cl}
\epsilon_{-}, & \text {if } \ell \text { is odd and } r \text { is even. } \\
\epsilon_{-}+\epsilon_{+}, & \text {if } \ell \text { and } r \text { are both odd. } \\
\epsilon_{+}, & \text {if } \ell \text { is even and } r \text { is odd. } \\
0, & \text { if } \ell \text { and } r \text { are both even. }
\end{array}\right.
$$

proof of part (iv) of Theorem [1. The hypothesis is that $\epsilon_{0}=0$, at least one of $\epsilon_{-}, \epsilon_{+}$is not zero and $\ell \equiv r \equiv 1(\bmod 2)$. Now $B$ has $e$ non-exceptional characters, of which $\ell+r$ are real-valued. So $e \equiv \ell+r$ is even. Then $b_{0}$ is not real, according to Corollary 12 , This in turn implies that $\nu_{0}=0$. So $\epsilon_{-}+\epsilon+=0$, according to (9). We conclude that $\epsilon_{-} \epsilon_{+}=-1$, which gives the conclusion of (iv).

\section{Passing From $B$ to its CANOnical Character}

Let $i=0, \ldots, a-1$. Then $N_{i}$ contains the normalizer $N_{0}$ of $D$ in $G$. So by Brauer's first main theorem there is a unique $p$-block $B_{i}$ of $N_{i}$ such that $B_{i}^{G}=B$. As $\left(B_{i}^{o}\right)^{G}=$ $B^{o}=B$, the uniqueness forces $B_{i}^{o}=B_{i}$. Now $B_{i}$ has defect group $D$ and inertial index $e=\left|E C_{i}: C_{i}\right|$. So $\ell\left(B_{a-1}\right)=e$ and $k\left(B_{a-1}\right)=e+\frac{p^{a}-1}{e}$. We first consider the block $B_{a-1}$ of the largest subgroup $N_{a-1}$. Following [D, Section 7], write

$$
\operatorname{IBr}\left(B_{a-1}\right)=\left\{\tilde{\chi}_{1}, \ldots, \tilde{\chi}_{e}\right\}, \quad \operatorname{Irr}\left(B_{a-1}\right)=\left\{\tilde{X}_{1}, \ldots, \tilde{X}_{e}\right\} \bigcup\left\{\tilde{X}_{\lambda} \mid \lambda \in \Lambda\right\},
$$

and set $\tilde{X}_{0}=\sum \tilde{X}_{\lambda}$.

Proposition 14. The exceptional characters in $B$ and $B_{a-1}$ have the same F-S indicators.

Proof. Suppose first that $|\Lambda| \geq 2$. According [D, (7.2)] there is a sign $d$ such that

$$
\left(\tilde{X}_{\lambda}-\tilde{X}_{\mu}\right)^{G}=d\left(X_{\lambda}-X_{\mu}\right), \quad \text { for all } \lambda, \mu \in \Lambda \text {. }
$$

It follows that $\left\langle\tilde{X}_{\lambda}, X_{\lambda}\right\rangle$ or $\left\langle\tilde{X}_{\mu}, X_{\lambda}\right\rangle$ is odd. So in view of part (i) of Theorem 1, the conclusion holds in this case.

From now on we suppose that $|\Lambda|=1$. Then $E$ has a single orbit on $\operatorname{Irr}(D)^{\times}$, which forces $|D|=p$ and $e=p-1$. As $\tilde{X}_{0}$ is the unique exceptional character in $B_{a-1}$, it is real valued. Then it follows from part (iii) of Theorem 1 that all real irreducible characters in $B_{a-1}$ have the same F-S indicators.

Now by [D, (7.3), (7.8), first two paragraphs of p40], there is a sign $\varepsilon_{0}^{\prime}$ such that

$$
\left(\tilde{X}_{0}-\sum_{i=1}^{p-1} \tilde{X}_{i}\right)^{G}=\varepsilon_{0}^{\prime} \sum_{i=0}^{p-1} \varepsilon_{i} X_{i} .
$$

Here $\varepsilon_{0}, \ldots, \varepsilon_{p-1}$ are as introduced earlier and $X_{0}$ can be chosen to be real, as $p$ is odd. Taking inner-products of characters, and reading modulo 2, we see that $\left\langle\tilde{X}_{i}^{G}, X_{0}\right\rangle$ is odd, for some real $\tilde{X}_{i}$. So $\epsilon\left(\tilde{X}_{i}\right)=\epsilon\left(X_{0}\right)$. Then by the previous paragraph $\epsilon\left(\tilde{X}_{0}\right)=\epsilon\left(X_{0}\right)$.

Proposition 15. All exceptional characters in $B_{0}, \ldots, B_{a-1}$ and $B$ have the same $F-S$ indicators. 
Proof. We prove this by induction on $|D|$. The base case $|D|=p$ holds, by Proposition 14. Suppose that $|D|>p$. We assume that the conclusion holds for all $p$-blocks with a cyclic defect group of order strictly less than $|D|$.

We use the bar notation for subgroups and objects associated with the quotient group $N_{a-1} / D_{a-1}$. Let $i=0, \ldots, a-1$. Then $\bar{N}_{i}$ is the normalizer of $\bar{D}_{i}$ in $\bar{N}_{a-1}$. As $C_{i}$ centralizes $D_{a-1}$, Theorem 5.8.11 of [NT] shows that $b_{i}$ dominates a unique block $\overline{b_{i}}$ of $\bar{C}_{i}$. Moreover $\bar{b}_{i}$ has cyclic defect group $\bar{D}$. Now $b_{i}$ has the unique irreducible Brauer character $\varphi_{i}$, and we can and do identify $\varphi_{i}$ with the unique irreducible Brauer character in $\bar{b}_{i}$. Then the inertia group of $\bar{b}_{i}$ in $\bar{N}_{i}$ is the inertia group of $\varphi_{i}$ in $\bar{N}_{i}$, which is $\overline{E C_{i}}$.

According to $\left[\mathrm{D}\right.$, Section 4], there is a unique $p$-block of $N_{i}$, denoted here by $\overline{B_{i}}$, which lies over $\overline{b_{i}}$. Moreover $\overline{B_{i}}$ has cyclic defect group $\bar{D}$. As inflation and induction of characters commute, this block is dominated by $B_{i}$. Now $B_{i}$ and $\overline{B_{i}}$ have the same inertial index as $\left|E C_{i}: C_{i}\right|=\left|\overline{E C_{i}}: \overline{C_{i}}\right|$. So by inflation $\operatorname{IBr}\left(\overline{B_{i}}\right)=\operatorname{IBr}\left(B_{i}\right)$. In particular $\overline{B_{i}}$ is the unique block of $\overline{N_{i}}$ that is dominated by $B_{i}$. Also by inflation $\operatorname{Irr}\left(\overline{B_{i}}\right) \subseteq \operatorname{Irr}\left(B_{i}\right)$.

As $|\bar{D}|<|D|$, all exceptional characters in $\bar{B}_{0}, \ldots, \bar{B}_{a-1}$ have the same F-S indicators, by our inductive hypothesis. But the inclusion $\operatorname{Irr}\left(\overline{B_{i}}\right) \subseteq \operatorname{Irr}\left(B_{i}\right)$ identifies the exceptional characters in $\bar{B}_{i}$ with exceptional characters in $B_{i}$. It now follows from part (i) of Theorem 1 that all exceptional characters in $B_{0}, \ldots, B_{a-1}$ have the same F-S indicators.

Recall that $b_{0}$ has a unique irreducible Brauer character $\varphi_{0}$. This is the canonical character of $B$, in the sense of [NT, 5.8.3]. For the next theorem, we simplify the notation of (44) for the irreducible characters in $b_{0}$ by writing $\chi_{\lambda}$ in place of $X_{0, \lambda}^{\prime}$, for all $\lambda \in \operatorname{Irr}(D)$. Then according to W. Reynolds [NT, 5.8.14], for $c \in C_{0}$ we have

$$
\chi_{\lambda}(c)= \begin{cases}\lambda\left(c_{p}\right) \varphi_{0}\left(c_{p}^{\prime}\right), & \text { if } c_{p} \in D . \\ 0, & \text { if } c_{p} \notin D .\end{cases}
$$

Then $\operatorname{Irr}\left(b_{0}\right)=\left\{\chi_{\lambda} \mid \lambda \in \operatorname{Irr}(D)\right\}$. Notice that $\chi_{1}$ is the unique irreducible character in $b_{0}$ whose kernel contains $D$.

Theorem 16. Suppose that $B$ has a real exceptional character. Then $N_{0} / C_{0}$ has a unique subgroup $T / C_{0}$ of order 2 , and all exceptional characters in $B$ have $F-S$ indicator equal to the Gow indicator $\epsilon_{T / C_{0}}\left(\chi_{1}\right)$.

Proof. Recall that $B$ has a real exceptional character if $b_{0}$ is real and $e$ is even, or if $b_{0}$ is not real and $e$ is odd. In both these cases $\left|N_{0}: C_{0}\right|$ is even. As $N_{0} / C_{0}$ is also cyclic, it has a unique subgroup $T / C_{0}$ of order 2 .

In view of Proposition [15, we may assume that $G=N_{0}$. So $B=B_{0}, D$ and $C_{0}$ are normal subgroups of $G$ and $E$ is the stabilizer of $b_{0}$ in $G$. Then $\Lambda$ is a set of representatives for the orbits of $N_{0}$ on $\operatorname{Irr}(D)^{\times}$. Set $E^{*}$ as the stabilizer of $\left\{b_{0}, b_{0}^{o}\right\}$ in $G$. Clifford correspondence defines a bijection between the irreducible characters of $E^{*}$ which lie over $b_{0}$ and the irreducible characters in $B$. This bijection preserves reality, and hence F-S indicators. So from now on we assume that $G=E^{*}$. 
As $\chi_{1}$ is invariant in $E$ and $E / C_{0}$ is cyclic, $\chi_{1}$ has $e$ extensions to $E$, which we denote by $\eta_{1}, \ldots, \eta_{e}$. Then $X_{i}:=\eta_{i}^{G}$, for $i=1, \ldots, e$, give the $e$ non-exceptional characters in $B$. Moreover $X_{\lambda}:=\chi_{\lambda}^{G}$, for all $\lambda \in \Lambda$, give the exceptional characters in $B$.

Following Corollary [12, there are three cases we must consider:

Case 1: $b_{0}$ is real, $e$ is even and $B$ has real non-exceptional characters. Then according to part (iii) of Theorem 1 all real irreducible characters in $B$ have the same F-S indicators. We choose notation so that $X_{1}$ is real. As $X_{1} \downarrow_{T}$ is a real extension of $\chi_{1}$ to $T$, it follows that $\epsilon\left(X_{1}\right)=\epsilon\left(X_{1} \downarrow_{T}\right)=\epsilon_{T / C_{0}}\left(\chi_{1}\right)$. This concludes Case 1 .

Case 2: $b_{0}$ is real, $e$ is even but $B$ has no real non-exceptional characters. As $\chi_{1}$ does not extend to a real character of $E$, it does not extend to a real character of $T$, according to Lemma 4. So $\epsilon_{T / C_{0}}\left(\chi_{1}\right)=-\epsilon\left(\chi_{1}\right)$, by the definition of the Gow indicator.

Now consider the notation used in the proof of part (i) of Theorem 11. Here $C_{i}=C_{0}$ and $\varphi_{i}=\varphi_{0}$ and $X_{i, 1}^{\prime}=\chi_{1}$, for $i=0, \ldots, a-1$. If $\lambda \in \Lambda$ then $\left(X_{\lambda}\right) \downarrow_{C_{0}}=\sum_{\tau \in G / C_{0}} \chi_{\lambda^{\tau}}$. So $d_{X_{\lambda}, \varphi_{i}}^{(x)}=\sum_{\tau \in G / C_{0}} \lambda\left({ }^{\tau} x\right)$, for all $x \in D^{\times}$. This means that $\varepsilon_{0} \gamma_{i}=1$, for $i=0, \ldots, a-1$. Now in (6), the term $\sigma$ is 0 , as none of $X_{1}, \ldots, X_{e}$ are real. So the first equation in (6) simplifies here to $-\epsilon\left(X_{\lambda}\right)=\epsilon\left(\chi_{1}\right)$, for all $\lambda \in \Lambda_{1}$. So $\epsilon\left(X_{\lambda}\right)=\epsilon_{T / C_{0}}\left(\chi_{1}\right)$, for all $\lambda \in \Lambda$, by the previous paragraph and Proposition 15.

Case 3: The final case is that $b_{0}$ is not real and $e$ is odd. As $B$ has an odd number $e$ of non-exceptional characters, at least one of them must be real valued. So we assume that $X_{1}$ is real. Then, just as in Case 1, all real irreducible characters in $B$ have the same F-S indicators.

As $\left|E: C_{0}\right|$ is odd and $|G: E|=2$, we have $G / C_{0}=E / C_{0} \times T / C_{0}$. Now $T / C_{0}$ conjugates $\operatorname{Irr}\left(b_{0}\right)$ into $\operatorname{Irr}\left(b_{0}^{o}\right)$. So $\chi_{1}$ is $T$-conjugate to $\bar{\chi}_{1}$. In particular $\chi_{1} \uparrow^{T}$ is irreducible and real valued. Now $X_{1}=\left(\eta_{1}\right) \uparrow^{G}$ and $\left(\eta_{1}\right) \downarrow_{C_{0}}=\chi_{1}$. So $\left(X_{1}\right) \downarrow_{T}=\left(\chi_{1}\right) \uparrow^{T}$, by Mackey's theorem.

Now from above $\epsilon\left(X_{\lambda}\right)=\epsilon\left(X_{1}\right)$, for all $\lambda \in \Lambda$. Also $\epsilon\left(X_{1}\right)=\epsilon\left(\left(X_{1}\right) \downarrow_{T}\right)$, as both are real valued. Finally $\epsilon\left(\left(X_{1}\right) \downarrow_{T}\right)=\epsilon_{T / C_{0}}\left(\chi_{1}\right)$, by the definition. This completes Case 3 .

Finally, we prove the application to ordinary characters as stated in the Introduction:

Proof of Theorem 2. Let $x$ be a weakly real $p$-element of $G$ of maximal order and set $Q:=\langle x\rangle$ and $N:=\mathrm{N}_{G}(Q)$. Let $\lambda$ be a faithful linear character of $Q$. Then $N_{\lambda}=\mathrm{C}_{N}(x)$ and $N_{\lambda}^{*}=\mathrm{C}_{N}^{*}(x)$. So $N_{\lambda}^{*}$ does not split over $N_{\lambda}$. By Lemma 3 there exists $\chi \in \operatorname{Irr}(N \mid \lambda)$ such that $\epsilon(\chi)=-1$.

Let $\tilde{B}$ be the $p$-block of $N$ which contains $\chi$ and let $D$ be a defect group of $\tilde{B}$. Then $Q \subseteq D$ and $\mathrm{N}_{G}(D) \subseteq N$. In particular $B:=\tilde{B}^{G}$ is defined and $B$ has defect group $D$. So $Q=D_{i}, N=N_{i}$ and $\tilde{B}=B_{i}$ for some $i \geq 0$, in cyclic defect group notation.

Notice that $\lambda$ is non-trivial. So $D \nsubseteq \operatorname{ker}(\chi)$. This means that $\chi$ is an exceptional character in $B_{i}$. So all exceptional characters in $B_{i}$, and hence also in $B$, are symplectic. The number of exceptional characters in $B$ is $\frac{|D|-1}{e}$, where $e$ is the inertial index of $B$. The number of weakly real $p$-conjugacy classes of $G$ is equal to the number of $N$-orbits on $Q^{\times}$, which equals $\frac{\left|D_{i}\right|-1}{\left|N_{i}: C_{i}\right|}$. As $\left|D_{i}\right| \leq|D|$ and $e \leq\left|N_{i}: C_{i}\right|$, we conclude that the 
number of symplectic irreducible characters of $G$ is not less than the number of weakly real $p$-conjugacy classes of $G$.

\section{Acknowledgement}

H. Blau and D. Craven alerted us to examples of real $p$-blocks which do not have a real irreducible character. We thank G. Navarro for permission to include his example. We had a number of interesting discussions with R. Gow on F-S indicators.

\section{REFERENCES}

[Al] J. L. Alperin Weights for Finite Groups, Proc. Symp. Pure Math. 47 (1987) 369-379.

[A] J. H. Conway, R. T. Curtis et al. ATLAS of finite groups: maximal subgroups and ordinary characters for simple groups, Clarendon Press, Oxford, 1985.

[B] R. Brauer, Some applications of the theory of blocks of characters of finite groups III, J. Algebra 3 (1966) 225-255.

[F] W. Feit, The representation theory of finite groups, North-Holland Math. Library 25, 1982.

[D] E. C. Dade, Blocks with cyclic defect groups, Ann. Math. 84 (1966) 20-48.

[GAP] The GAP Group, GAP - Groups, Algorithms, and Programming, Version 4.8.8; 2017, (https://www.gap-system.org).

[G] R. Gow, Real valued and 2-rational group characters, J. Algebra 61 (1979) 388-413.

[Gr] J. A. Green, Walking around the Brauer Tree, J. Austral. Math. Soc. 17 (1974) 197-213.

[M1] J. Murray, Strongly real 2-blocks and the Frobenius-Schur indicator, Osaka J. Math. 43 (1) (2006) 201-213.

[M2] J. Murray, Components of the involution module in blocks with a cyclic or Klein-four defect group J. Group Theory 11 (1) (2008) 43-62.

[M3] J. Murray, Real subpairs and Frobenius-Schur indicators of characters in 2-blocks, J. Algebra 322 (2) (2009) 489-513.

[NT] H. Nagao, Y. Tsushima, Representations of finite groups, Academic Press, 1987.

$[\mathrm{W}]$ W. Willems, Duality and forms in representation theory, Representation theory of finite groups and finite-dimensional algebras (Bielefeld, 1991), Progr. Math., 95, Birkhuser, Basel, 1991, 509-520.

Department of Mathematics and Statistics, National University of Ireland Maynooth, IRELAND

E-mail address: John.Murray@maths.nuim.ie 